\title{
Mid-term Patient Reported Outcomes and Survivorship Following Robotic Assisted Total Knee Replacement: A Cohort Study
}

\author{
Eric A. Chen ${ }^{1}$, Adil Husain ${ }^{2}$, Nicholas Billow ${ }^{3}$, \\ Jan A. Koenig ${ }^{1}$, and Christopher Plaskos ${ }^{4}$ \\ ${ }^{1}$ NYU Winthrop University Hospital, Long Island, NY, USA \\ ${ }^{2}$ St. Louis University School of Medicine \\ ${ }^{3}$ Renaissance School of Medicine at Stony Brook Hospital \\ ${ }^{4}$ Omnilife Science, Boston, USA
}

\begin{abstract}
Despite the advantages of real time alignment assessment and visual feedback while balancing offered by robotic assisted total knee arthroplasty, few clinical studies have reported patient outcomes. The purpose of this study is to report the midterm patient reported outcomes and survivorship of a computer-navigated TKA system with a robotic cutting guide. This patient cohort is the first IRB approved series of patients treated in the United States with this robotic knee system. This study serves as a midterm follow-up study on for this cohort, upon which learning curve, intra-operative efficiency, and deformity management were previously reported

Recipients of 152 consecutive total knee arthroplasties using a computernavigated TKA system performed by a single surgeon between June 2010 and January 2012 were surveyed between 5-7 years post-operatively. 94 patients were reachable for outcome measures and survivorship data was obtained in 98 patients. Mean patient age at follow up was $74.6+/-8.6$ years. Implant survivorship was $99.0 \%$ at an average of 6.5 years. Mean patient reported knee outcome scores were 62.7 (KSS-SF) and 79.5 (KOOS-JR). Overall satisfaction rate was reported as "satisfied or very satisfied" in $80.2 \%$, "neutral" in $11.0 \%$, and "dissatisfied or very dissatisfied" in $7.7 \%$ of patients.

Robotic assisted total knee arthroplasty using a computer-navigated TKA system with a robotic cutting guide appears to provide a durable outcome with sustainable midterm patient reported outcomes and excellent survivorship. Further follow up is required to determine if there are long term outcome and survivorship benefits of robotic assisted total knee arthroplasty.
\end{abstract}


Mid-term Patient Reported Outcomes and Survivorship Following Robotic Assisted ... E. Chen et al.

\section{Introduction}

Total knee arthroplasty (TKA) has become one of the most effective treatments for the chronic pain of end stage degenerative arthritis of the knee that is refractory to conservative modes of treatment. Most recently, technologic advancements have allowed the use of computer navigation and robotics in total knee arthroplasty with real time virtual planning, robotic assisted execution, instantaneous validation of the bone cuts, as well as evaluation of pre and post-surgical kinematic alignment and balance. This has allowed for improvement in the consistency and the accuracy of component placement and limb alignment compared to conventional methods [1]. Because postoperative alignment has been shown to correlate with patient satisfaction following total knee arthroplasty [5], technological improvements that allow us to better restore limb alignment may in turn lead to better patient functional outcomes.

Despite the advantages of real time alignment assessment and visual feedback and confirmation of soft tissue balancing offered by robotic assisted total knee arthroplasty, few clinical studies have reported patient outcomes following computer and robotic assisted total knee arthroplasty. Most recent comparisons between computer-assisted and conventional total knee arthroplasty suggest that computer-assisted technique provides better pain relief and restored better function compared to conventional TKA at two years [6].

The purpose of this study is to report the midterm patient reported outcomes and survivorship of a computer-navigated TKA system with a robotic cutting guide. This patient cohort is the first IRB approved series of patients treated in the United States with this robotic knee system. This study serves as a midterm follow-up study for this cohort, upon which learning curve, intra-operative efficiency, and deformity management were previously reported [4].

\section{Methodology}

Recipients of the first 152 consecutive computer assisted total knee arthroplasties performed by a single surgeon between June 2010 and January 2012 were identified and contacted between 5-7 years post-operatively following institutional IRB approval for the study. All total knee arthroplasties in the cohort were performed using the OMNIBotics computer-navigated TKA system with robotic cutting guide and APEX total knee arthroplasty implants. A detailed description of the post-operative alignment of the cohort has previously been published [4]. Patient demographics were obtained via retrospective chart review. Patients that were reachable via phone for follow up were surveyed using the Knee Society Score - Short Form (KSS-SF), Knee Injury and Osteoarthritis Outcome Score for Joint Replacement (KOOS-JR), Veterans RAND 12 Item Health Survey (VR-12), and for implant survivorship. Implant survivorship alone was recorded for patients who were unable to complete the outcomes survey due to mental or physical ailments.

\section{Results}

94 patients were reachable for outcome measures and 98 patients were reached for survivorship data. One patient provided an incomplete set of outcome data. 11 patients were known to have been deceased. Mean patient age at follow up was $74.6+/-8.6$ years. There was a $58 \%$ female to $42 \%$ male ratio. Implant survivorship was $99.0 \%$ at an average of 6.5 years, with one revision performed out of 98 patients due to aseptic loosening of a tibial baseplate following trauma. There were no incidents of prosthetic joint infection in the cohort. Mean patient reported knee outcome 
scores were 62.7 +/- 18.9 (KSS-SF) and 79.5 +/- 18.2 (KOOS-JR). Mean VR-12 scores were 51.3 +/1 12.5 for mental health and $42.2+/-12.2$ for physical health. Overall satisfaction rate was reported as $91.2 \%$ and overall dissatisfaction rate was reported as $7.7 \%$.

\begin{tabular}{|lcc|}
\hline Outcome Score & mean & SD \\
\hline KSS-SF - Symptoms Pain Walking & 1.2 & 2.1 \\
KSS-SF - Symptoms Pain Stairs & 2.0 & 2.6 \\
KSS-SF - Symptoms Normal & 4.0 & 1.5 \\
KSS-SF - Symptoms Total (25 pts max) & $\mathbf{2 0 . 8}$ & $\mathbf{5 . 3}$ \\
& & \\
KSS-SF - Satisfaction Light Household & 6.5 & 2.0 \\
\% Neutral, Satisfied, or Very Satisfied & $\mathbf{9 1 . 2 \%}$ & \\
\% Dissatisfied or Very Dissatisfied & $\mathbf{7 . 7 \%}$ & \\
*single patient with incomplete KSS-SF data & & \\
& & \\
KSS-SF - Function Walk Score (20 max) & 13.3 & 6.8 \\
KSS-SF - Activities Walk (15 max) & 12.3 & 3.7 \\
KSS-SF - Activities Stairs (15 max) & 12.2 & 3.3 \\
KSS-SF - Activities Rising From Chair (15 max) & 12.3 & 4.0 \\
KSS-SF - Activities Running (20 max) & 2.7 & 6.1 \\
KSS-SF - Score Discretionary (15 max) & 10.4 & 5.4 \\
TOTAL KSS Short Form activity score (100 max) & $\mathbf{6 2 . 7}$ & 18.9 \\
& & \\
KOOS-JR & $\mathbf{7 9 . 5}$ & 18.2 \\
& & \\
VR-12 Mental Score & & \\
VR-12 Physical Score & 51.3 & 12.5 \\
VR-12 Supplement Physical & 42.2 & 12.2 \\
VR-12 Supplement Emotional & 3.1 & 1.0 \\
Survivorship at 6.5 years (mean) follow-up & 3.1 & 0.9 \\
\hline
\end{tabular}

\section{Discussion}

Computer assisted total knee arthroplasty using a computer-navigated TKA system with a robotic cutting guide appears to provide a durable outcome with sustainable midterm patient reported outcomes and excellent survivorship at 5-7 years post operatively. It was noted that because the ability to run was a significant component in the calculation of the KSS-SF score, the functional performance of this aging cohort may have been negatively skewed. However, overall satisfaction rates were high at $80.2 \%$ and neutral at $11.0 \%$ in our cohort.

This study is limited in that $33 \%$ of patients were lost to follow up. This was significantly higher than studies of similar length follow up [7] likely due to the high propensity of retirees in the area to relocate secondary to the cost of living in this particular geographic region. Recent studies show that from 2010-2017, New York State experienced negative net domestic migration of over 1 million persons. Long Island alone experienced a negative net domestic migration of 90,000 persons over this period [8]. The high attrition rate of New Yorkers, especially those in search of lower cost of 
Mid-term Patient Reported Outcomes and Survivorship Following Robotic Assisted ... E. Chen et al.

living post-retirement may have played a contributing role in challenges in establishing mid to longterm follow up.

Although previous studies have shown marginal improvement in patient reported outcomes in the short term with computer assisted surgery compared to conventional methods [2], distinctions in functional outcome may not begin to manifest themselves until more remote follow up. Recent literature suggests that limb alignment plays a significant role in patient functional outcomes [5] and that navigation guided total knee arthroplasty can be a valuable tool in management large preoperative coronal and sagittal deformities [3]. As such, further follow up is required to determine if there are long term outcome and survivorship benefits of computer and robotic assisted total knee arthroplasty.

\section{Reference}

1. Cheng T, Zhao S, Peng X, Zhang X. Does computer-assisted surgery improve postoperative leg alignment and implant positioning following total knee arthroplasty? A meta-analysis of randomized controlled trials? Knee Surgery, Sports Traumatology, Arthroscopy.

2011;20(7):1307-22.

2. Gøthesen Ø, Espehaug B, Havelin LI, Petursson G, Hallan G, Strøm E, et al. Functional outcome and alignment in computer-assisted and conventionally operated total knee replacements. The Bone \& Joint Journal. 2014;96-B(5):609-18.

3. Koenig JA, Plaskos C. Influence of Pre-Operative Deformity on Surgical Accuracy and Time in Robotic-Assisted TKA. Bone \& Joint Journal. 2013; 95-B (S-28) 62.

4. Koenig JA, Suero EM, Plaskos C. Surgical accuracy and efficiency of computer-navigated TKA with a robotic cutting guide-report on the first 100 cases. J Bone Joint Surg Br. 2012;94-B(S)-XLIV:103.

5. Matsuda S, Kawahara S, Okazaki K, Tashiro Y, Iwamoto Y. Postoperative Alignment and ROM Affect Patient Satisfaction After TKA. Clinical Orthopaedics and Related Research ${ }^{\circledR}$. 2012;471(1):127-33.

6. Petursson G, Fenstad AM, Gøthesen Ø, Dyrhovden GS, Hallan G, Röhrl SM, et al. Computer-Assisted Compared with Conventional Total Knee Replacement. The Journal of Bone and Joint Surgery. 2018;100(15):1265-74.

7. Scott CEH, Bell KR, Ng RT, Macdonald DJ, Patton JT, Burnett R. Excellent 10-year patientreported outcomes and survival in a single-radius, cruciate-retaining total knee arthroplasty. Knee Surgery, Sports Traumatology, Arthroscopy. 2018;27(4):1106-15.

8. Vink JK. 2017 County and Economic Development Regions Population Estimates Analysis of the US Census Bureau Vintage 2017 Total County Population Estimates [Internet]. 2017 [cited 2019Mar]. Available from: https://pad.human.cornell.edu/papers/downloads/Vintage 2017 report.pdf 